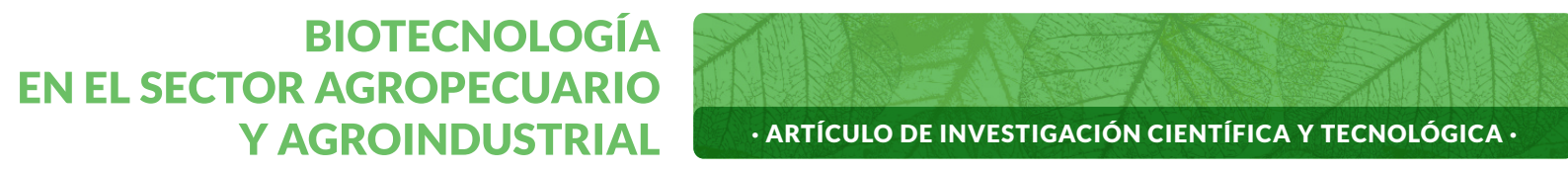

Vol. 20 No 1 · Enero-Junio 2022 · ISSN-1692-3561· ISSN-e 1909-9959 · DOI: https://doi.org/10.18684/rbsaa.v19.n2.2021.1879

\title{
Obtención de "Snacks" de piña (Ananas comosus) mediante las técnicas combinadas de Ventana de Refractancia y Fritura con Aire Caliente*
}

\section{Obtaining "Snacks" of pineapple (Ananas Comosus) by the combined techniques of Refractance Window and Hot Air Frying"}

\author{
GONZÁLEZ-HOYOS, LINA-VANESSA ${ }^{1}$; ARROYO-RINCÓN, ANA-MARÍA²; TOBAR-SUAREZ, ALEXANDER³; \\ POLANÍA-RIVERA, ANNA-MARÍA4; AYALA-APONTE, ALFREDO5; DÍAZ-ORTIZ, ALBERTO ${ }^{6}$
}

Historial del artículo

Recibido para evaluación: 11 de Abril 2021

Aprobado para publicación: 11 de Octubre 2021

* Título del proyecto de origen: "Incremento de la competitividad sostenible en la agricultura de ladera en todo el departamento, Valle del Cauca, Occidente". Financiación: Sistema General de Regalías. Culminación: marzo 2021.

1 Universidad del Valle, Facultad de Ingeniería, Escuela de Ingeniería de Alimentos. Ingeniera de Alimentos. Cali, Colombia.

2 Universidad del Valle, Facultad de Ingeniería, Escuela de Ingeniería de Alimentos. Ingeniera de Alimentos. Cali, Colombia.

3 Universidad del Valle, Facultad de Ingeniería, Escuela de Ingeniería de Alimentos. Ingeniero de Alimentos. Cali, Colombia.

4 Universidad del Valle, Facultad de Ingeniería, Escuela de Ingeniería de Alimentos. Estudiante de Doctorado en Ingeniería con énfasis en Ingeniera de Alimentos. Cali, Colombia. https://orcid.org/0000-0001-9646-2266

5 Universidad del Valle, Facultad de Ingeniería, Escuela de Ingeniería de Alimentos. Ph.D. Ciencia y Tecnología de los Alimentos. Cali, Colombia. https://orcid.org/0000-0003-0310-3577

6 Universidad del Valle, Facultad de Ingeniería, Escuela de Ingeniería de Alimentos. Ph.D. Ingeniería de Procesos. Cali, Colombia. https://orcid.org/0000-0003-0310-3577 


\section{RESUMEN}

Las técnicas de secado de Ventana de Refractancia (VR) y Fritura con Aire Caliente (AF), permiten obtener snacks de frutas de alta calidad nutricional y fisicoquímica. El propósito de este trabajo fue evaluar las técnicas de secado de WR-AF para obtener "snacks" de piña deshidratada. Se emplearon trozos de piña de la variedad MD2 con geometría triangular ( $40 \mathrm{~mm}$ de lado y $4 \mathrm{~mm}$ de espesor). En el secado por WR se emplearon tres temperaturas $\left(70,80\right.$ y $\left.90^{\circ} \mathrm{C}\right)$. Para cada temperatura se modeló la cinética de secado mediante cuatro modelos matemáticos (Newton, Page; Wang \& Singh, y Midilli). Se determinó: color, en términos de Luminosidad ( $\left.L^{*}\right)$ y cambio total de color $(\Delta E)$, actividad de agua $\left(a_{w}\right)$, coeficientes de difusión y energía de activación. Para las muestras secadas en la combinación de las técnicas VR-AF, se determinaron las curvas de secado, $L^{*}, \Delta E, a_{w} y$ vitamina $C$. En total se realizaron 4 tratamientos en el secado con $A F$ a $100^{\circ} \mathrm{C}$; tres de las cuales fueron muestras tratadas (MT) previamente por WR, y el otro fue muestra no tratada (MNT). Los resultados mostraron que, a mayor temperatura de secado por WR mayor fue el coeficiente de difusión y menor fue el tiempo de secado. Para un contenido de humedad de $9 \%$ (d.b), los tratamientos a 90,80 y $70^{\circ} \mathrm{C}$ requirieron 90,110 y 130 min de secado, respectivamente. El modelo de Midilli fue el que mejor ajustó las cinéticas de secado. Se observó que las técnicas de VR-AF permitieron obtener menor tiempo de secado, mayor retención de vitamina $C$ y de color respecto a las MNT. Estos resultados indican que la técnica WR-AF es una alternativa viable para producir "snacks" de piña en tiempos mas cortos y con mayor conservación de las características de calidad.

\section{ABSTRACT}

Refractance Window (WR) and Hot Air-Frying (HAF) dried techniques, allows to obtain high nutritional and physicochemical quality fruit snacks. The aim of this work consisted on evaluating WR-AF dry techniques to obtain dehydrated pineapple snacks. MD2 variety pineapple pieces were cut into triangular shapes with $40 \mathrm{~mm}$ by side and $4 \mathrm{~mm}$ in thickness.

For WR drying, three temperatures were set $\left(70,80\right.$ and $\left.90^{\circ} \mathrm{C}\right)$. For each one, a dry-out kinetics model was applied adopting 4 math models (Newton, Page; Wang \& Singh, and Midilli). Also, color as in Luminosity ( $\left.L^{*}\right)$, total color changes $(\Delta E)$, water activity $\left(a_{w}\right)$, diffusion coefficient, and activation energy were determined. On dried samples under WR-HAF mixed method, drying curves were determined, as well as, $L^{*}, \Delta E, a_{w}$ and vitamin $C$. In total, four treatments were evaluated in the AF process at $100^{\circ} \mathrm{C}$; three of which were treated sam-

Cómo citar este artículo: GONZÁLEZ-HOYOS, LINA-VANESSA; ARROYO-RINCÓN, ANA-MARÍA; TOBAR-SUAREZ, ALEXANDER; POLANÍA-RIVERA, ANNA-MARÍA; AYALA-APONTE, ALFREDO; DÍAZ-ORTIZ, ALBERTO. Obtención de "Snacks" de piña (Ananas comosus) mediante las técnicas combinadas de Ventana de Refractancia y Fritura con Aire Caliente. Revista Biotecnología en el Sector Agropecuario y Agroindustrial, v. 20, n. 1, 2022, p. 165-178. Doi: https://doi.org/10.18684/rbsaa.v19. n2.2021.1879

\section{PALABRAS CLAVE:}

Snacks, Piña; Cinética; Secado; Ventana de refractancia; Fritura con aire caliente; Actividad de agua; Color; Vitamina C.

\section{KEYWORDS:}

Snacks; Pineapple; Kinetics; Drying; Refractance Window; Air-Frying; Water activity; Color; Vitamin C. 
ples (TS) under WR, and one sample left untreated (UNTS). Results showed that the higher the drying temperature under WR, the greater the diffusion coefficient and lower the drying time. To reach $9 \%$ (d.b) of humidity, treatments at 90 , 80 and $70{ }^{\circ} \mathrm{C}$ required 90,110 and 130 min, respectively. Midilli model adjusted better to drying kinetics. WR-HAF techniques allowed less drying time, higher vitamin C, and color retention compared to UNTS. Results demonstrated that combined WR-HAF is a feasible alternative to obtain pineapple snacks in shorter time while preserving high quality characteristics.

\section{INTRODUCCIÓN}

La piña (Ananas comosus) es originaria de Brasil y Paraguay y es una de las frutas que más se consume en el mundo (Olmos, 2015) y apetecida por su sabor, aroma y aporte nutricional; se puede consumir en fresco o como producto procesado tipo refrescos, enlatados dulces, entre otros (Wu \& Shiau, 2015; Rani \& Tripathy, 2019). De acuerdo con estimaciones de la Organización de las Naciones Unidas para la Agricultura y la Alimentación (FAO), la piña está en segundo lugar en la producción mundial de frutas tropicales, con 28,3 millones de toneladas en 2018 (Braga et al., 2020). A pesar de que se evidencian diversos productos procesados a bse de piña, son pocos los desarrollos de "snacks" como producto deshidratado con textura crujiente y con alta retención de calidad. Por otro lado, la piña, como en las frutas en general, presenta corto tiempo de vida útil por su alto contenido de agua o actividad de agua, por lo que es necesario aplicar nuevos procesos para desarrollar productos con alta retención de calidad y, a la vez, incrementar la vida útil. El secado es una alternativa como método de conservación de los alimentos, ya que extiende la vida útil (Braga et al., 2020), reduce el contenido de humedad, disminuyendo el peso y el volumen y facilitando el transporte y almacenamiento de productos, además, se disminuye la actividad del agua por debajo de 0,6, inhibiendo los microorganismos de deterioro, así como los cambios físicos y químicos durante el almacenamiento (Vega-Gálvez et al., 2007).

Para su conservación, la piña se ha deshidratado por diversos métodos como el secado con aire caliente, el secado solar, la deshidratación osmótica, la liofilización, las microondas, entre otros (Salazar et al., 2019; Mohammed et al., 2020; Zzaman et al., 2021), sin embargo, estos métodos evidencian algunos efectos negativos en la calidad fisicoquímica y nutricional en los alimentos. El secado con aire caliente es la técnica más utilizada en la industria alimentaria, pero los tiempos de operación son relativamente prolongados y las características del producto final no siempre son deseables (Zhang et al., 2018), mientras que el secado por liofilización presenta productos con alta retención de calidad, pero su inconveniente radica en que emplea largos tiempos de procesamiento (mayor de 24 h), alto consumo de energía y, en consecuencia, altos costos de operación.

En los últimos años se han desarrollado métodos de secado con alta retención de la calidad de frutas y hortalizas, estos son el secado por Ventana de Refractancia (VR) y la técnica de Fritura con Aire Caliente (FAC) llamado también "Air Frying". La técnica de secado por VR fue desarrollada por MCD Technologies, Inc., es relativamente simple, barata y se puede aplicar a una variedad de productos. La VR utiliza agua como medio de calentamiento a una temperatura inferior al punto de ebullición colocando el producto sobre una película plástica MAYLAR que es relativamente transparente a la radiación infrarroja del agua (Ortiz-Jerez et al., 2015; Raghavi et al., 2018). En varias investigaciones se ha demostrado que alimentos secados por VR obtienen productos con alta retención en calidad nutricional, fisicoquímica y organoléptica en comparación con otros métodos de secado en diversos vegetales (Nemzer et al., 2018; Leiton-Ramírez et al., 2020). El método por FAC es una técnica de secado convectivo con aire caliente de alta velocidad que puede usarse con o sin adición de aceite al producto. Este método le confiere al producto agradables propiedades texturales y sensoriales; es una nueva técnica con la que se obtienen productos alimenticios en cortos tiempos, logrando un producto saludable, deshidratado y con textura crujiente (Shaker, 2015). A pesar de que con estas técnicas (VR y FAC) se obtienen alimentos secos con retención significativa de calidad, la combinación de ellas permite obtener snacks con menores tiempos de proceso y mayor retención de calidad al compararla con una sola técnica. El objetivo de este estudio fue obtener Snacks de piña mediante el método combinado de las técnicas de VR y FAC, evaluando cinéticas de secado, cambios de color, $\mathrm{a}_{w}$ y vitamina $\mathrm{C}$. 


\section{MÉTODO}

La experimentación se realizó en los laboratorios de la Escuela de Ingeniería de Alimentos de la Universidad del Valle (Cali, Valle del Cauca, T: $28^{\circ} \mathrm{C}$ y $70 \%$ humedad relativa). Se emplearon frutos de piña MD2 proporcionados por productores de piña del norte del mismo departamento; con condiciones de procesamiento de $24^{\circ} \mathrm{C}$ y $85 \%$ $\mathrm{HR}$, las piñas se lavaron con agua potable, se pelaron y se cortaron en trozos regulares con geometría triangular (40 $\mathrm{mm}$ de lado y $4 \mathrm{~mm}$ de espesor)

\section{Secado por ventana de refractancia}

Los trozos de piña se secaron en un equipo piloto de VR (CEI-ROBOTS, Col) que consiste en un tanque lleno de agua (largo: 0,6 m, ancho: 0,4 m) en cuya superficie se coloca una membrana de poliéster transparente (tipo Mylar $\left.{ }^{\mathrm{TM}}\right)$. Se emplearon tres temperaturas para el calefactor de agua $\left(70,80\right.$ y $\left.90^{\circ} \mathrm{C}\right)$. Las muestras se colocaron sobre la membrana de poliéster (Mylar) para el secado y, a diferentes tiempos, se retiraron muestras para construir la curva de secado graficando el contenido de humedad (g agua/g materia seca) vs el tiempo de proceso (min) (Ochoa-Martínez et al., 2012). Los experimentos se realizaron por triplicado.

El contenido de humedad (CH) se determinó de acuerdo con el método 20.013 (AOAC, 1980). La actividad del agua $\left(a_{w}\right)$ se midió con el equipo Decagón (AquaLab CX-1, USA) con una sensibilidad de 0,001. El color se determinó mediante el uso de un espectrocolorímetro (HunterLab, USA), obteniendo las coordenadas de color $L^{*} a^{*}$, $\mathrm{b}^{*}$ del sistema $\mathrm{CIE}-\mathrm{L}^{*} \mathrm{a}^{*} \mathrm{~b}^{*}$ a partir de los espectros de reflexión de las muestras, utilizando como referencia el iluminante D65 y el observador $10^{\circ}$ : a partir de estas coordenadas se calcularon las diferencias de color $(\Delta \mathrm{E})$ (Ecuación 1) (Villamizar et al., 2012). Como variables de respuesta indirectas en el proceso por VR se calcularon los coeficientes de difusión del agua y la energía de activación. El coeficiente de difusión se calculó usando el primer término de la solución analítica de la segunda ley de Fick (Ecuación 2). Se asumió que la geometría es una placa plana infinita, que la distribución de la humedad inicial es uniforme, la resistencia a la transferencia de masa despreciable y no existe encogimiento (Onwude et al., 2016).

$$
\Delta E=\sqrt{\left(L^{*}-L_{0}^{*}\right)^{2}+\left(a^{*}-a_{0}^{*}\right)^{2}+\left(b^{*}-b_{0}^{*}\right)^{2}}
$$

Dónde $\Delta E$ : Es la diferencia de color; $L^{*}$ es el valor de $L^{*}$ para la muestra tratada; $L_{0}^{*}$ es valor de $L^{*}$ para la muestra fresca; $a^{*}$ es el valor de a* para la muestra tratada; $a_{0}^{*}$ es el valor de a* para la muestra fresca; $b^{*}$ es el valor de $b^{*}$ para la muestra tratada; $b_{0}^{*}$ es el valor de $b^{*}$ para la muestra fresca.

$$
M R=\frac{\left(M_{t}-M_{e}\right)}{\left(M_{0}-M_{e}\right)}=\frac{8}{\pi^{2}} \sum_{n=1}^{\infty} \frac{1}{(2 n+1)^{2}} \exp \left(-D_{e} \frac{(2 n+1)^{2} t \pi^{2}}{4 l^{2}}\right)
$$

Dónde $M R$ Es la relación adimensional de humedad; los subíndices, $0, t$ y $e$ representan las humedades en el tiempo $0, t$ y en el equilibrio respectivamente; $n$ es el número de términos de la sumatoria, $D e$ es el coeficiente difusivo del agua, $l$ es la dimensión característica de la muestra (Ochoa-Martínez \& Ayala-Aponte, 2005).

Para el cálculo de la difusividad efectiva $(D$ ) se linealizó la ecuación 3 convirtiéndose en la ecuación 4; la pendiente de la ecuación se igualó a $-D_{\mathrm{e}} \pi^{2} / 4 l^{2}$ dónde $l$ es el espesor medio de la muestra (Vega et al., 2007). 


$$
\begin{aligned}
& M R=\frac{8}{\pi^{2}} \exp \left(\frac{-D_{e} \pi^{2} t}{4 l^{2}}\right) \\
& \ln (M R)=\ln \frac{8}{\pi^{2}}-\left(\frac{D_{e} \pi^{2}}{4 l^{2}}\right) t
\end{aligned}
$$

La energía de activación se determinó mediante la ecuación 5 tipo Arrhenius. La dependencia de $D_{e}$ con la temperatura de secado por VR se determinó empleando esta ecuación tipo Arrhenius (Onwude et al., 2016). La ecuación 5 se linealizó y se convirtió en ecuación 6; la pendiente de la ecuación se igualó a $-E_{a} / R$ y se despejó la Energía de activación $\left(E_{a}\right)$.

$$
\begin{aligned}
& D_{e}=D_{o} \exp \left(\frac{-E_{a}}{R T}\right) \\
& \ln \left(D_{e}\right)=\frac{1}{T}\left(\frac{-E_{a}}{R}\right)+\ln \left(D_{o}\right)
\end{aligned}
$$

Dónde: $E a$ es la energía de activación [J/mol]; es la constante de los gases [J/mol K]; es la temperatura [K]; $D_{o}$ es una constante.

Los valores experimentales de las cinéticas de secado por VR se ajustaron mediante 4 modelos matemáticos: Newton, Page, Wang \& Singh y Midilli (Michalewicz et al., 2011; Zhang et al., 2017) (Cuadro 1). El mejor modelo cinético se empleó en las tres temperaturas para predecir los tiempos de secado en las muestras de piña para alcanzar un nivel de humedad de $45 \%$ (b,h) o su equivalente en base seca de $81,82 \%$; estas muestras parcialmente deshidratadas a 70,80 y $90^{\circ} \mathrm{C}$ se secaron por la técnica de FAC.

Cuadro 1. Modelos cinéticos para el ajuste de valores experimentales de humedad en el secado de rodajas de piña MD2 por la técnica de VR.

Nombre
Newton
Page
Wang y Singh
Midilli

$$
\begin{aligned}
& M R=\exp (-k t) \\
& M R=\exp \left(-k t^{n}\right) \\
& M R=1+A t+B t^{2} \\
& M R=A \cdot \exp \left(-k t^{n}\right)+B t
\end{aligned}
$$

\section{Modelo}

\section{Secado por la técnica de fritura con aire caliente}

Se empleó un equipo de FAC a $100{ }^{\circ} \mathrm{C}$ con una capacidad de 4,2 L (Imusa, easy fry), este puede secar con o sin presencia de aceite. Consta con un control análogo de temperatura y tiempo. Se emplearon dos niveles de humedad de trozos de piña, un nivel fue $0,45 \mathrm{~g}$ agua/g $\mathrm{mt}$; donde $\mathrm{mt}=$ masa total $(0,8182 \mathrm{~g}$ agua/g ms; donde $\mathrm{ms}=$ materia seca) obtenido en cada temperatura $\left(70,80\right.$ y $\left.90^{\circ} \mathrm{C}\right)$ en el secado por VR; estas muestras son llamadas muestras tratadas (MT) y el otro nivel fue 0,86 $\pm 0,02 \mathrm{~g}$ agua/g m.t (6,14 g agua/g ms) correspondiente a la humedad de la piña en estado fresco como muestra no tratada por VR (MNT). Se obtuvieron 4 tratamientos para el secado por FAC, correspondientes a las MT $\left(70,80\right.$ y $90^{\circ} \mathrm{C}$ ) y MNT (piña fresca o muestra control). Se realizaron las curvas de secado de piña en los cuatro tratamientos por FAC. A diferentes tiempos se determinó el $\mathrm{CH}$ para obtener la curva de secado, $y$ al final del secado ( $1 \% \mathrm{bs}$ ) se midieron el color, $\mathrm{a}_{\mathrm{w}}$, y vitamina $\mathrm{C}$. El diseño se realizó completamente al azar en los 4 tratamientos. Los experimentos se realizaron por triplicado. Los resul- 
tados se analizaron mediante el programa estadístico SPSS 18 (versión 23) y se realizó un análisis de varianza (ANOVA) con un nivel de significancia del $95 \%$.

El contenido de vitamina $C$ se determinó por reflectometría, que es una técnica basada en la interacción entre la materia y la luz (Poveda-Nuñez, 2014). Se utilizó un refractómetro digital (Rqflex 10 Merck; Reflectoquant, EDM Millipore Corp., Darmstadt, Germany), diluyendo $1 \mathrm{~g}$ de muestra en $10 \mathrm{~mL}$ de agua destilada. El resultado se expresó como $\mathrm{mL}$ de ácido ascórbico por cada $100 \mathrm{~g}$ de muestra (Ecuación 11). El análisis se realizó por triplicado para cada muestra (Domínguez et al., 2016). La determinación de la vitamina C se realizó a las muestras frescas y a las muestras secas al final del experimento por los dos métodos (VR y FAC).

$\frac{m g \text { de ácido ascórbico }}{100 \mathrm{~g} \text { muestra }}=\frac{\text { Lectura } * \text { volumen } \mathrm{H}_{2} \mathrm{O} \text { destilada }}{10 * \text { Peso de la muestra }}$

Para el cálculo de la pérdida de vitamina C, se estimó mediante la diferencia entre la concentración final e inicial de la muestra, divida por la muestra inicial

\section{RESULTADOS}

\section{Curvas de secado por VR}

La Figura 1 muestra las curvas de secado de piña secadas por VR. El CH de las muestras frescas de piña fue de $6,14 \pm 0,12 \mathrm{~g}$ agua/g $\mathrm{ms}(0,86 \pm 0,02 \mathrm{~g}$ agua/g $\mathrm{mt}), y \mathrm{a}_{\mathrm{w}}$ de 0,9815 $\pm 0,0043$., valores similares a los reportados por otros investigadores (Salazar et al., 2019; Ashutosh et al., 2021). Se evidenció un efecto significativo ( $<<0,05)$ de la temperatura de secado por VR sobre el contenido de humedad en la piña: a mayor temperatura, menor fue el tiempo de proceso para obtener un mismo nivel de humedad, o caso contario, a mayor temperatura mayor pérdida de humedad se obtuvo para un mismo tiempo de secado en las tres temperaturas. Diferentes autores reportan resultados similares en distintos alimentos como en batata biofortificada (Souza et al., 2019), en cubos de piña (Sethi \& Kaur, 2019) y en cáscaras de manzana (Moussaoui et al., 2021).

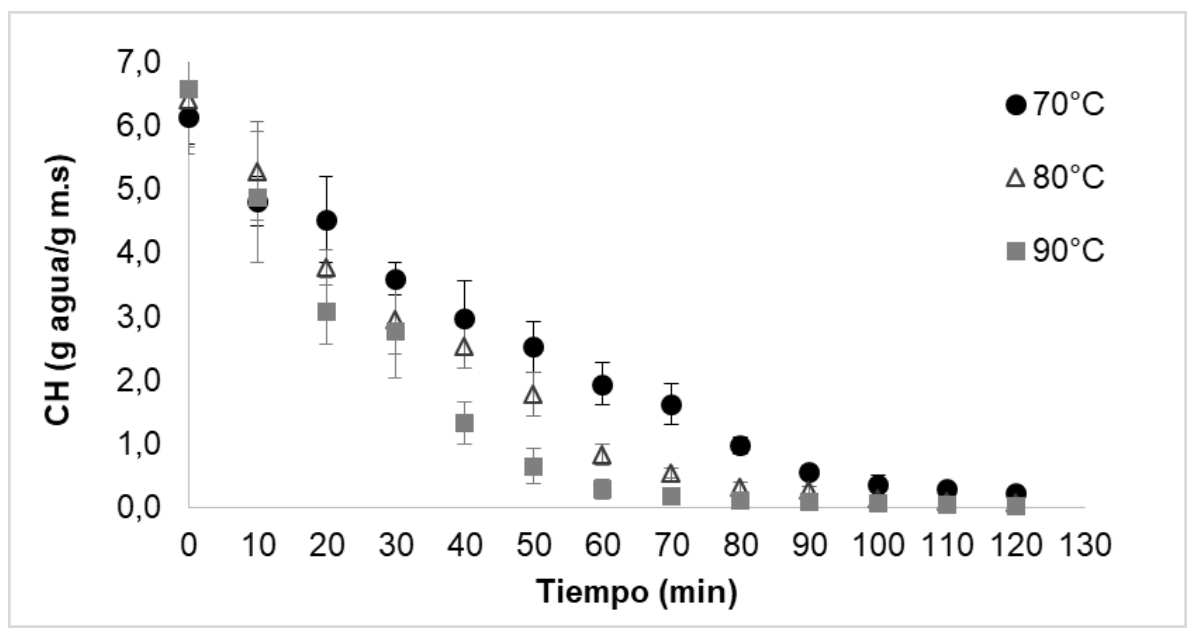

Figura 1. Cinéticas de secado de muestras de piña mediante VR a 70,80 y $90^{\circ} \mathrm{C}$. 
Para obtener un $\mathrm{CH}$ aproximado de $9 \%$ (b.s), se observa que a $90^{\circ} \mathrm{C}$ se alcanza en el menor tiempo con 90 min, seguido de $80^{\circ} \mathrm{C}$ con $110 \mathrm{~min}$ y $70^{\circ} \mathrm{C}$ con $130 \mathrm{~min}$. Este resultado puede explicarse, ya que al incrementarse la temperatura se aumenta la transferencia de calor, la tasa de evaporación y la migración de agua desde interior hacia la superficie del alimento, ocasionando que la muestra pierda agua en menor tiempo (Souza et al., 2019; Macedo et al., 2020).

Es evidente que con la técnica de VR se alcanzan significativamente tiempos más cortos respecto a otros métodos de secado de piña como el secado convectivo (Salazar et al., 2019; Mohammed et al., 2020) Estos tiempos más cortos en VR comparado con otros métodos, pueden ser atribuidos a la combinación de los mecanismos de transferencia de calor como la radiación (energía infrarroja del agua), la conducción en la membrana plástica y en el alimento y al aumento de la convección por efecto de la circulación del aire en las muestras que están en la superficie de la membrana plástica.

\section{Coeficiente de Difusión y energía de activación}

Los coeficientes de difusión efectiva $\left(D_{e}\right)$ del agua en el secado por VR se incrementaron significativamente $(p<0,05)$ con el aumento de la temperatura, obteniéndose valores de $7,75 \times 10^{-10}, 1,708 \times 10^{-09}$ y 2,027 X10-09 $\mathrm{m}^{2} / \mathrm{s}$, respectivamente, para 70,80 y $90^{\circ} \mathrm{C}$. Estos valores de $D_{e}$ están dentro del rango general de $10^{-11}-10^{-09}$ $\mathrm{m}^{2} / \mathrm{s}$ para el secado de alimentos (Macedo et al., 2020) y tienen el mismo orden de magnitud según los reportados en otros estudios en piña (Ponkham et al., 2012; Berbert et al., 2016). También, coincide con el secado de otras frutas como en banano (Macedo et al., 2020) y mora (Kaveh et al., 2020) El incrementó de $D_{e}$ con el aumento de la temperatura puede explicarse por la disminución de la viscosidad del agua permitiendo su difusión molecular en los capilares del producto, aumentando el valor de difusividad de humedad (Corrêa et al., 2010), comportamiento que también ha sido re portado en batata (Souza et al., 2019) y en piña (Silva et al., 2015; Berbert et al., 2016).

La energía de activación ( $\mathrm{E}_{\mathrm{a}}$ ) para el proceso de secado por VR en las tres temperaturas fue de 50,03 kJ/mol. Este valor se encuentra dentro del rango de valores encontrados en la literatura para alimentos de 12,7 a 110 $\mathrm{kJ} / \mathrm{mol}$ (Montes et al., 2008). La energía de activación representa la energía requerida para iniciar la difusión de la humedad del alimento durante el secado (Olanipekun et al., 2015): otros autores encontraron un valor relativamente similar de $\mathrm{E}_{\mathrm{a}}(41,53 \mathrm{~kJ} / \mathrm{mol})$ en el secado de banano en bandejas (Macedo et al., 2020).

\section{Modelación de las cinéticas de secado}

El Cuadro 2 contiene los parámetros cinéticos estimados mediante 4 modelos (Newton, Page, Wang \& Singh y Midilli), incluyendo los criterios de calidad $\left(\mathrm{R}^{2}\right), \mathrm{E}(\%), \mathrm{RMSE}$ y $\left(\mathrm{x}^{2}\right)$ para la evaluación del ajuste. Los resultados mostraron que todos los modelos presentaron buen ajuste; sin embargo, el modelo Midilli fue el que mejor ajustó los valores experimentales en las tres temperaturas de secado por presentar valores de $\mathrm{R}^{2}$ más próximos a 1,0 y RMSE y $x^{2}$ más cercanos a cero (Figura 2). Algunos investigadores han reportado que el modelo de Midilli modela con precisión el comportamiento del secado en varias frutas como el banano (Macedo et al., 2020) y la piña (Onwude et al., 2016). 
Cuadro 2. Parámetros cinéticos de los modelos Newton, Page, Wang \& Singh, Midilli para muestras de piña secadas por el método de VR.

\begin{tabular}{|c|c|c|c|c|c|c|}
\hline Modelo & Temperatura $\left({ }^{\circ} \mathrm{C}\right)$ & Coeficientes modelo & $\mathbf{R}^{2}$ & $E(\%)$ & RMSE & $x^{2}$ \\
\hline \multirow{3}{*}{ Newton } & 70 & $\mathrm{k}: 1,2140$ & 0,9739 & 0,4471 & 0,0492 & 0,0004 \\
\hline & 80 & $\mathrm{k}: 1,6810$ & 0,9807 & 0,8040 & 0,0445 & 0,0006 \\
\hline & 90 & $\mathrm{k}: 2,2710$ & 0,9820 & 0,8453 & 0,0421 & 0,0007 \\
\hline \multirow{3}{*}{ Page } & 70 & $\mathrm{k}: 1,2280 \mathrm{n}: 1,2220$ & 0,9849 & 0,2382 & 0,0374 & 0,0009 \\
\hline & 80 & $\mathrm{k}: 1,8210 \mathrm{n}: 1,2570$ & 0,9928 & 0,2124 & 0,0272 & 0,0002 \\
\hline & 90 & $\mathrm{k}: 2,6160 \mathrm{n}: 1,2420$ & 0,9906 & 0,2804 & 0,0305 & 0,0001 \\
\hline \multirow{3}{*}{$\begin{array}{l}\text { Wang \& } \\
\text { Singh }\end{array}$} & 70 & A: $-0,1934$ B:-0,0761 & 0,8073 & 0,5123 & 0,1336 & 0,0361 \\
\hline & 80 & A: $-0,4876$ B:0,0740 & 0,8122 & 0,9904 & 0,1890 & 0,0780 \\
\hline & 90 & A: $-0,7317$ B:0,2081 & 0,8347 & 0,8537 & 0,1276 & 0,0292 \\
\hline \multirow{3}{*}{ Midilli } & 70 & k:0,9235 n:1,0150 A:0,9841 B: -0,0748 & 0,9918 & 0,1896 & 0,0276 & 0,0000 \\
\hline & 80 & k:1,7230 n:1,2140 A:0,9960 B: -0,0113 & 0,9934 & 0,1561 & 0,0256 & 0,0000 \\
\hline & 90 & $\mathrm{k}: 2,5850 \mathrm{n}: 1,2460 \mathrm{~A}: 0,9920 \mathrm{~B}:-0,0021$ & 0,9907 & 0,4313 & 0,0303 & 0,0000 \\
\hline
\end{tabular}

Se observa que el parámetro cinético k aumenta con el incremento de la temperatura, lo que indica dependencia con la temperatura; similar comportamiento se observó en el secado de Aloe vera (Jha et al., 2016; Ayala et al., 2021). Esta dependencia con la temperatura indica que $\mathrm{k}$ se puede asociar con la pérdida de agua de la muestra, considerándolo como un parámetro pseudo-difusivo (Ah-Hen et al., 2013).

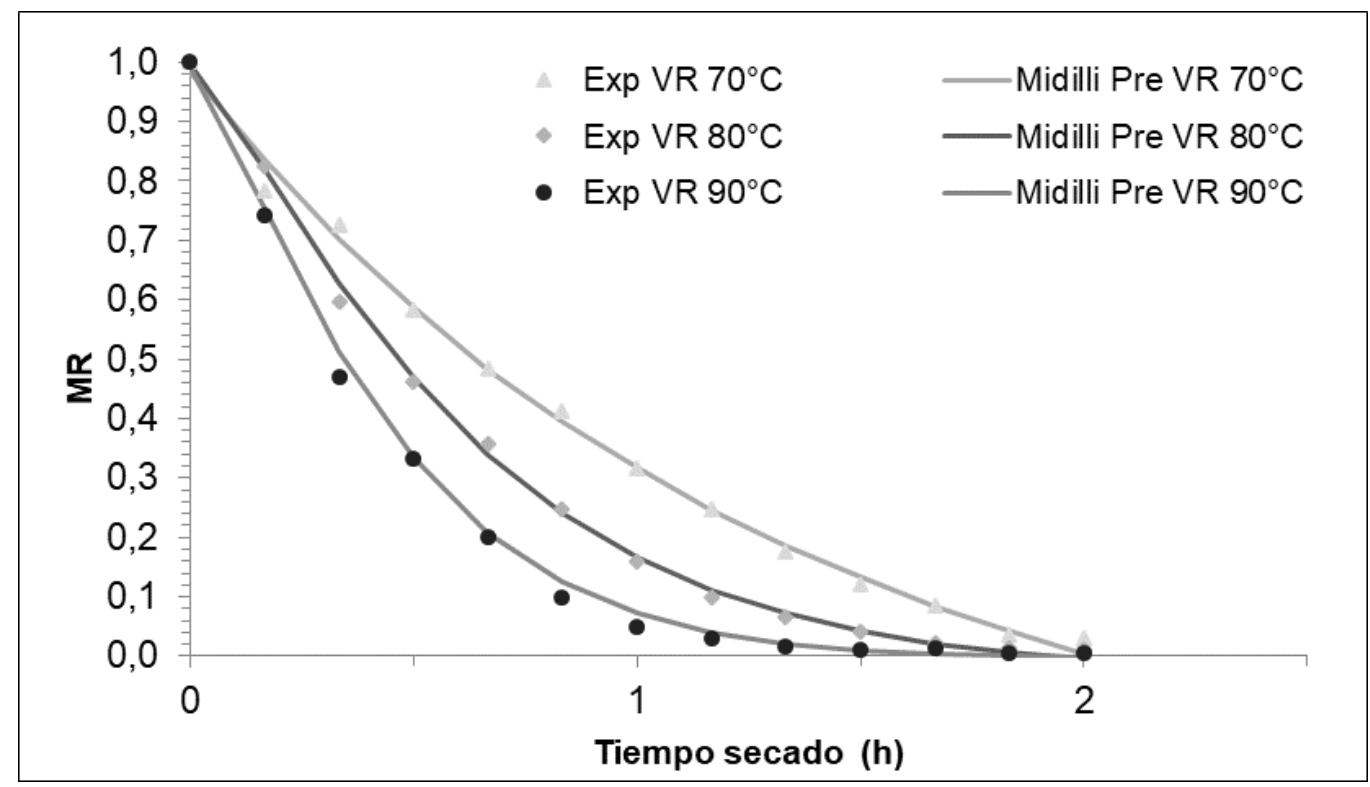

Figura 2. Valores de la relación de humedad predicha por el modelo Midilli frente a la relación de humedad experimental.

Sustituyendo los parámetros del Cuadro 2 (modelo Midilli) y la relación de humedad (MR) en la Ecuación 9 del Cuadro 1, se encontraron los tiempos para las tres temperaturas en el que las muestras de piña alcanzan el CH de $45 \%$ b.h. o su equivalente en base seca de $81,82 \%$. Los tiempos alcanzados con este modelo de midilli en las tres temperaturas $\left(70,80\right.$ y $\left.90^{\circ} \mathrm{C}\right)$ fueron respectivamente 90,67 y 50 min. 


\section{Color y actividad del agua en muestras de piña con una humedad de 0,45 g agua/ g mt (0,8182 g agua/g ms)}

Del proceso de secado por VR en las tres temperaturas, se obtuvieron muestras de piña con un $\mathrm{CH}$ de $45 \%$ (b.h), como pretratamiento al secado por FAC. En el Cuadro 3 se presentan los cambios de color y $a_{w}$ de muestras de piña en estado fresco y secadas por VR con $45 \%$ (b.h) de humedad. Se observa una disminución de luminosidad y del cambio total de color en las tres temperaturas de secado desde el inicio hasta el nivel de humedad $45 \%$. Este resultado puede ser atribuido a la temperatura de proceso en la fruta, ya que la piña es susceptible al pardeamiento no enzimático por su alto contenido de azúcares (Zzaman et al., 2021). Sin embargo, al comparar entre los tres pretratamientos, no se observó un efecto significativo $(p>0,05)$ del nivel de temperatura sobre el color (no se evidenciaron efectos en $L^{*}$ ni en $\Delta E$ ); este comportamiento puede estar asociado por presentar el mismo nivel de humedad entre los pretratamientos.

Cuadro 3. Valores de color y $a_{w}$ para diferentes condiciones de deshidratación en piña MD2 secadas a un $\mathrm{CH}$ de $45 \%$ (b.h).

\begin{tabular}{|c|c|c|c|c|c|c|}
\hline \multirow{2}{*}{ Tratamiento } & \multicolumn{4}{|c|}{ Color } & \multicolumn{2}{c|}{$\mathrm{a}_{\mathrm{w}}$} \\
\cline { 2 - 7 } & \multicolumn{2}{|c|}{$\mathrm{L}^{*}$} & \multicolumn{2}{c|}{$\Delta \mathrm{E}$} & \multicolumn{2}{c}{} \\
\hline $70^{\circ} \mathrm{C}$ & 43,88 & $\pm 2,19^{\mathrm{a}}$ & 6,26 & $\pm 0,14^{\mathrm{a}}$ & 0,524 & $\pm 0,053^{\mathrm{a}}$ \\
\hline $80^{\circ} \mathrm{C}$ & 42,25 & $\pm 1,85^{\mathrm{a}}$ & 7,35 & $\pm 0,52^{\mathrm{a}}$ & 0,522 & $\pm 0,019^{\mathrm{a}}$ \\
\hline $90^{\circ} \mathrm{C}$ & 42,21 & $\pm 2,16^{\mathrm{a}}$ & 7,67 & $\pm 0,35^{\mathrm{a}}$ & 0,515 & $\pm 0,088^{\mathrm{a}}$ \\
\hline
\end{tabular}

Letras diferentes indican una diferencia significativa entre los diferentes tratamientos $(p<0,05)$.

Por otro lado, se observó que la $a_{w}$ en los pretratamientos disminuyó significativamente $(p<0,05)$ desde 0,987(estado fresco) hasta valores que variaron entre 0,515 y 0,522, debido a la pérdida de agua durante el secado, ya que la $a_{w}$ decrece con la disminución del CH. Estos valores de $a_{w}$ indican que las muestras con $45 \%$ (b.h) son seguras durante el almacenamiento frente al ataque de microorganismos por presentar valores inferiores a la $a_{w}$ crítica de 0,63 (Ochoa-Martínez et al., 2012; Sethi \& Kaur, 2019). Igual que en el color, no se evidenció entre los pretratamientos un efecto significativo $(p>0,05)$ de la temperatura sobre la $a_{w}$, comportamiento que se esperaba, ya que los pretratamientos presentan similar contenido de humedad.

\section{Secado con tecnología de Fritura con Aire Caliente}

En la Figura 3 se observan las curvas de secado de piñas tratadas y no tratadas durante el proceso de deshidratación por la técnica de $\mathrm{FAC}$ a $100^{\circ} \mathrm{C}$. El CH inicial de las muestras de piña MNT fue de 0,8541 g agua $/ \mathrm{mt}(5,856$ $\pm 0,18 \mathrm{~g}$ agua/g ms), mientras que el $\mathrm{CH}$ inicial para las $\mathrm{MT}$ fue de $0,4500 \pm 0,003 \mathrm{~g}$ agua/g $\mathrm{mt}(0,8182 \mathrm{~g}$ agua/g $\mathrm{ms})$, obtenida previamente en el proceso por $\operatorname{VR}\left(70,80\right.$ y $\left.90^{\circ} \mathrm{C}\right)$.

Las MT presentaron significativamente $(p<0,05)$ menor contenido de agua con respecto a la MNT (control) para un mismo tiempo de secado; de forma contraria, puede decirse que las MT alcanzaron menor tiempo de secado respecto a la MNT para obtener un mismo nivel de humedad. Puede notarse que para alcanzar un $\mathrm{CH}$ del $1 \%$ (b.s), las MT alcanzaron menores tiempos entre 18 y 24 min, mientras que la MNT alcanzó 45 min, resultado esperado ya porque las MT inician con menor contenido de humedad respecto a la MNT. 


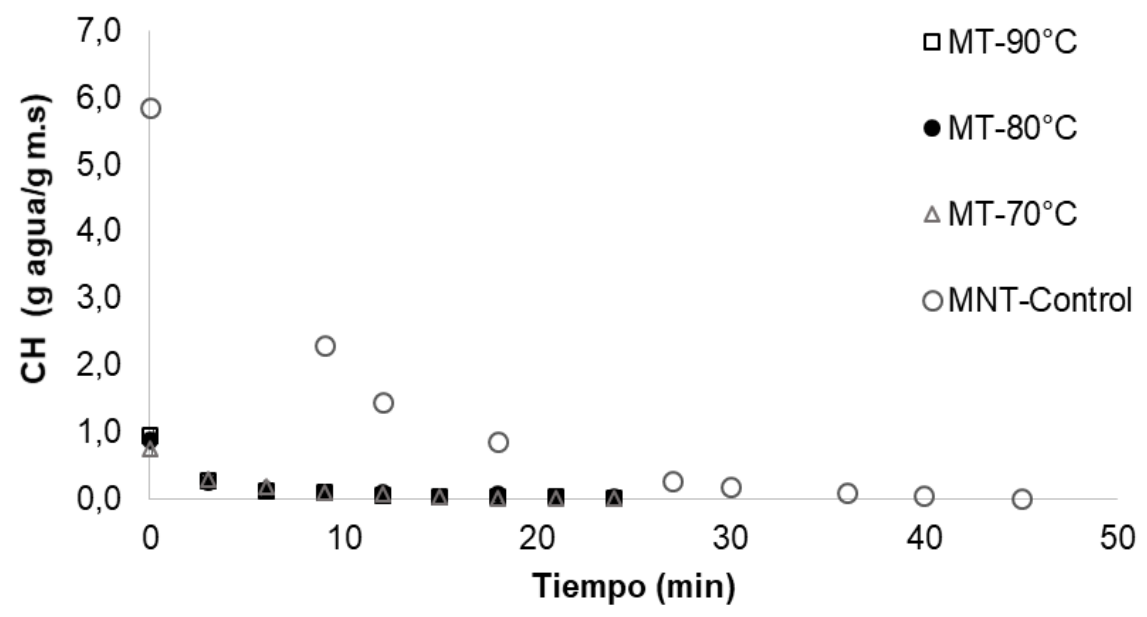

Figura 3. Cinéticas de secado de muestras de piña tratada y no tratada durante el proceso por AF a $100^{\circ} \mathrm{C}$.

Con respecto al $\mathrm{CH}$, entre las MT no presentaron diferencias significativas $(p>0,05)$ durante el secado por FAC, lo que indica posiblemente que la temperatura $\left(70,80\right.$ y $90^{\circ} \mathrm{C}$ ) en el secado por VR no ocasionó cambios significativos en la estructura de la fruta y, por consiguiente, no afectó la salida del agua durante el proceso de FAC. Este resultado también puede estar influenciado por contener el mismo nivel de humedad inicial (45 \% b.h), permitiendo la salida del agua a una misma velocidad en los tres pretratamientos.

En el Cuadro 4 se presentan los cambios de color, $a_{w}$, y vitamina $C$ de muestras de piña secadas a $100^{\circ} \mathrm{C}$ por la técnica de FAC para un $\mathrm{CH}$ de $1 \%$ (b.s). La piña en estado fresco (MNT) presenta las siguientes características: $45,02 \pm 0,88$ de luminosidad ( $\left.L^{*}\right), 0,9815 \pm 0,0043$ de $a_{w}$ y $358,37 \pm 2,35 \mathrm{mg} / 100 \mathrm{~g} \mathrm{~m} . \mathrm{s}$ de vitamina $\mathrm{C}$.

Cuadro 4. Valores de color, $\mathrm{a}_{\mathrm{w}}$ contenido y pérdida de Vitamina $\mathrm{C}$ para muestras de piña MD2 secadas por FAC hasta un CH de $1 \%$ (b.s).

\begin{tabular}{|c|c|c|c|c|c|c|c|c|c|}
\hline \multirow{3}{*}{$\begin{array}{l}\text { Tratamiento } \\
\text { MNT (Control) } \\
\end{array}$} & \multicolumn{4}{|c|}{ Color } & \multirow{2}{*}{\multicolumn{2}{|c|}{$a_{w}$}} & \multirow{2}{*}{\multicolumn{2}{|c|}{$\begin{array}{c}\text { Contenido de vit C } \\
\text { (mg/100 g m.s) }\end{array}$}} & \multirow{3}{*}{$\begin{array}{c}\text { Pérdida de Vit } \\
\text { C (\%) } \\
52,27 \pm 0,92^{\mathrm{a}}\end{array}$} \\
\hline & \multicolumn{2}{|c|}{$\mathbf{L}^{*}$} & \multicolumn{2}{|c|}{$\Delta \mathrm{E}$} & & & & & \\
\hline & 30,04 & $\pm 1,21^{a}$ & 19,57 & $\pm 0,02^{\mathrm{a}}$ & 0,195 & $\pm 0,008^{a}$ & 171,06 & $\pm 13,80$ & \\
\hline $\mathrm{MT}-70^{\circ} \mathrm{C}$ & 37,21 & $\pm 2,41^{b}$ & 13,57 & $\pm 0,34^{\mathrm{b}}$ & 0,209 & $\pm 0,018^{a}$ & 212,69 & $\pm 26,85$ & $40,65 \pm 0,88^{b}$ \\
\hline $\mathrm{MT}-80^{\circ} \mathrm{C}$ & 36,98 & $\pm 1,83^{b}$ & 14,09 & $\pm 0,22^{\mathrm{b}}$ & 0,198 & $\pm 0,046^{a}$ & 224,84 & $\pm 32,65$ & $37,25 \pm 0,97^{b}$ \\
\hline $\mathrm{MT}-90^{\circ} \mathrm{C}$ & 35,94 & $\pm 1,69^{b}$ & 14,78 & $\pm 0,67^{\mathrm{b}}$ & 0,184 & $\pm 0,039^{a}$ & 220,11 & $\pm 24,61$ & $38,58 \pm 0,54^{b}$ \\
\hline
\end{tabular}

Letras diferentes indican una diferencia significativa entre los diferentes tratamientos $(p<0,05)$.

Se presentó un efecto significativo $(p<0,05)$ entre las MT y las MNT sobre la luminosidad y el cambio total de color, mientras que entre las MT no se presentaron diferencias significativas $(p>0,05)$. Puede notarse que la MNT presentó el menor valor de $L^{*}$ con 30,04 $\pm 1,21$ y el mayor cambio de color $\Delta \mathrm{E}$ con $19,57 \pm 0,02$, lo que indica que presentó mayor oscurecimiento y, en consecuencia, mayor cambio de color; este comportamiento puede ser atribuido al mayor tiempo de proceso durante el secado por FAC, con 45 min para obtener un $\mathrm{CH}$ de $1 \%$ (b.s); mientras que las MT alcanzaron aproximadamente $21 \mathrm{~min}$ para este mismo nivel de humedad. Se ha evidenciado que el tiempo de secado influye en el cambio de color; de acuerdo con Izli et al. (2018), reportan mayor tiempo de proceso en el secado convectivo a 80 y $90^{\circ} \mathrm{C}$ con menores valores de $L^{*}$ respecto al secado por microondas de muestras de piña. Este comportamiento se ha presentado en otras frutas como en la pera (Marzec et al., 2020) y en la manzana (Rajoriya et al., 2020). 
La $a_{w}$ no mostró diferencias significas $(p<0,05)$ en los cuatro tratamientos, varió en valores entre 0,195 y 0,209. Estos valores cercanos de $a_{w}$, pueden ser atribuidos por tener un similar $\mathrm{CH}$. Los resultados de actividad de agua indican que los "snacks" de piña secados por la técnica de FAC son altamente estables contra los microorganismos durante el almacenamiento, ya que presentan valores alejados de la $a_{w}$ crítica de 0,63 (Ochoa-Martínez et al., 2012; Sethi \& Kaur, 2019).

Con respecto a la vitamina $C$, se presentaron diferencias significativas $(p<0,05)$ entre las MT y MNT al final del secado para un $\mathrm{CH}$ de $1 \%$ (b.s). La MNT o control (solo secada por FAC) presentó mayor pérdida de vitamina C frente a las muestras tratadas y posteriormente secadas por FAC; esta mayor pérdida de vitamina $C$ en la MNT $(52,27 \%)$ puede asociarse al mayor tiempo $(45 \mathrm{~min})$ de exposición al secado a $100^{\circ} \mathrm{C}$. Se ha evidenciado que a mayor tiempo de secado se provocan mayores pérdidas o menor retención de vitaminas en frutas (Marín et al., 2006). Este resultado significa que la reducción de humedad en las MT o método combinado (VR-FAC) mostró un efecto protector frente a la pérdida de vitamina $\mathrm{C}$ comparada con la muestra no tratada. Entre las muestras tratadas $\left(70,80\right.$ y $\left.90^{\circ} \mathrm{C}\right)$ no se evidenciaron diferencias significativas $(p>0,05)$ sobre la pérdida de vitamina $\mathrm{C}$, alcanzando valores relativamente cercanos que variaron entre 37,25 y 40,65\%. Este comportamiento puede ser atribuido al menor tiempo de exposición de las muestras durante el secado por FAC a $100^{\circ} \mathrm{C}$, ya que contenían menor agua al inicio del proceso, secadas previamente por VR. De acuerdo con Ayala et al. (2021), la pérdida de vitamina C en los procesos de secado en alimentos se asocia con el hecho de que es uno de los nutrientes más sensibles al calor y a los largos tiempos de secado, por consiguiente, la combinación de estos dos métodos de secado (VR-FAC) permite retener significativamente este nutriente en los snacks de piña por el tiempo corto de secado.

\section{CONCLUSIONES}

La técnica de ventana de refractancia demostró obtener tiempos cortos de proceso y mayor coeficiente de difusión del agua con el incremento de la temperatura de secado. El modelo predictivo de Midilli fue el que mejor ajustó las curvas de secado por ventana de refractancia en las tres temperaturas, técnica que presenta un alto potencial como alternativa de conservación de piña, ya sea como pretratamiento o como técnica directa.

Como pretratamiento al proceso de FAC se observa que la VR es efectiva para mantener las propiedades de color y vitamina C en los "snacks" de piña." Se evidencia que muestras de piña pretratadas con VR (con menor contenido de humedad) y posteriormente secadas por la técnica de FAC presentan menores tiempos de secado, mayor retención de la vitamina $\mathrm{C}$ y en el color (mayor claridad y menor cambio total de color). Estos resultados demuestran que el secado de piña por el método combinado de VR seguido de la técnica de FAC puede ser una alternativa para obtener un producto tipo "snacks" de piña con tiempos cortos de procesamiento y alta retención de características de color y vitamina C.

\section{AGRADECIMIENTOS}

Al Sistema General de Regalías con el proyecto de "Incremento de la competitividad sostenible de la agricultura de ladera en todo el departamento, Valle del Cauca, occidente". Universidad Nacional - Sede Palmira. Centro Internacional de Agricultura Tropical (CIAT). Universidad del Valle - Sede Cali. 


\section{REFERENCIAS}

AH-HEN, K.; ZAMBRA, C.E.; AGUËRO, J.E.; VEGA-GÁLVEZ, A.; LEMUS-MONDACA, R. Moisture Diffusivity Coefficient and Convective Drying Modelling of Murta (Ugni molinae Turcz): Influence of Temperature and Vacuum on Drying Kinetics. Food and Bioprocess Technology, v. 6, n. 4, 2013, p. 919-930. https://doi.org/10.1007/s11947-011-0758-5

AOAC. Official Methods of Analysis, 13th ed. Association of the Official Agricultural Chemists. Washington D.C. (United States of America): 1980, p. 376-384.

ASHUTOSH, S.C.; SHWETA, S.; ATUL, D.; POWAR. Optimization of pineapple drying based on energy consumption, nutrient retention, and drying time through Multi-Criteria Decision-Making. Journal of Cleaner Production, v. 292, 2021, 135907. https://doi.org/10.1016/j.jclepro.2021.125913

AYALA, A.; CARDENAS, J.D.; TIRADO, D.F. Aloe vera gel drying by Refractance Window: Drying kinetics and High-Quality Retention. Foods, v. 10, 2021, n. 1445, p. 1-16. https://doi.org/10.3390/foods10071445

BERBERT, P.A.; TEREZINHA, M.; DE OLIVEIRA, R.; MARTINAZZO, A.P. Drying of Pineapple Slices in Natura and Pre- Osmodehydrated in Inverted Sugar, v. 32, n. 3, 2016, p. 597-610. https://doi.org/10.14393/BJ-v32n3a2016-26201

BRAGA, V.; GUIDI, L.R.; DE SANTANA, R.C.; ZOTARELLI, M.F. Production and characterization of pineapplemint juice by spray drying. Powder Technology, v. 375, 2020, p. 409-419. https://doi.org/10.1016/j.powtec.2020.08.012

CORRÊA, P.C.; OLIVEIRA, G.H.H.; BOTELHO, F.M.; GONELI, A.L.D.; CARVALHO, F.M. Modelagem matemática e determinação das propriedades termodinâmicas do café (Coffea arabica L.) durante o processo de secagem. Revista Ceres, v. 57, n. 5, 2010, p. 595-601. https://doi.org/10.1590/S0034-737X2010000500005

DOMINGUEZ, P.; MEDINA, J.J.; MIRANDA, L.; LÓPEZ, J.M; ARIZA, M.T.; SORIA, C.; SANTOS, B.; TORRES, E.A.; HERNANDEZ, I. Effect of Planting and Harvesting Dates on Strawberry Fruit Quality under High Tunnels. International Journal of Fruit Science, v. 16, n.1, 2016, p. 228-238. https://doi.org/10.1080/15538362.2016.1219291

IZLI, N.; IZLI, G.; TASKIN, O. Impact of different drying methods on the drying kinetics, color, total phenolic content and antioxidant capacity of pineapple. Journal of Food, v. 16, n. 1, 2018, p. 213-221. https://doi.org/ 10.1080/19476337.2017.1381174

JHA, R.K.; PRABHAKAR, P.K.; SRIVASTAV, P.P.; RAO, V.V. Influence of temperature on vacuum drying characteristics, functional properties and micro structure of Aloe vera (Aloe barbadensis Miller) gel. Research in Agricultural Engineering, v. 61, n. 4, 2016, p. 141-149. https://doi.org/10.17221/13/2014-RAE

KAVEH, M.; TAGHINEZHAD, E.; AZIZ, M. Effects of physical and chemical pretreatments on drying and quality properties of blackberry (Rubus spp.) in hot air dryer. Food Science and Nutrition, v. 8, n. 7, 2020, p. 3843-3856. https://doi.org/10.1002/fsn3.1678

LEITON-RAMIIREZ, Y.M.; AYALA-APONTE, A.; OCHOA-MARTÍNEZ, C.I. Physicochemical Properties of Guava Snacks as Affected by Drying Technology. Processes, v. 8, n. 1, 2020, p. 106. https://doi.org/10.3390/pr8010106

MACEDO, L.L.; VIMERCATI, W.C.; DA SILVA-ARAÚJO, C.; SARAIVA, S.H.; TEIXEIRA, L.J.Q. Effect of drying air temperature on drying kinetics and physicochemical characteristics of dried banana. Journal of Food Process Engineering, v. 43, n. 9, 2020, p. 1-10. https://doi.org/10.1111/jfpe.13451

MARÍN, B.E.; LEMUS, M.R.; FLORES, M.V.; VEGA, G.A. La rehidratación de alimentos deshidratados. Revista Chilena de Nutrición, v. 33, n. 3, 2006, p. 527-538. https://doi.org/10.4067/S0717-75182006000500009

MARZEC, A.; KOWALSKA, H.; KOWALSKA, J.; DOMIAN, E.; LENART, A. Influence of Pear Variety and Drying Methods on the Quality of Dried Fruit. Molecules (Basel, Switzerland), v. 25, n. 21, 2020. https://doi.org/10.3390/molecules25215146 
MICHALEWICZ, J.S.; HENRIQUEZ, J.R.; CHARAMBA, J.C. Secado de Cajuil (Anacardium Occidentale L.): Estudio Experimental y Modelado de la Cinética de Secado. Información Tecnológica, v. 22, n. 6, 2011, p. 63-74. https://doi.org/10.4067/S0718-07642011000600007

MOHAMMED, S.; EDNA, M.; SIRAJ, K. The effect of traditional and improved solar drying methods on the sensory quality and nutritional composition of fruits: A case of mangoes and pineapples. Heliyon, v. 6, n. 6, 2020. https://doi.org/10.1016/j.heliyon.2020.e04163

MONTES, E.J.; TORRES, R.; ANDRADE, R.D.; PÉREZ, O.A.; MARIMON, J.L.; MEZA, I.I. Modelado de la cinética de secado de ñame (Dioscorea rotundata) en capa delgada. Ingenieria e Investigacion, v. 28, n. 2, 2008, p. 45-52.

MOUSSAOUI, H.; BAHAMMOU, Y.; TAGNAMAS, Z.; KOUHILA, M.; LAMHARRAR, A.; IDLIMAM, A. Application of solar drying on the apple peels using an indirect hybrid solar-electrical forced convection dryer. Renewable Energy, v. 168, 2021, p. 131-140. https://doi.org/10.1016/j.renene.2020.12.046

NEMZER, B.; VARGAS, L.; XIA, X.; SINTARA, M.; FENG, H. Phytochemical and physical properties of blueberries, tart cherries, strawberries, and cranberries as affected by different drying methods. Food Chemistry, v. 262, 2018, p. 242-250. https://doi.org/10.1016/j.foodchem.2018.04.047

OCHOA-MARTÍNEZ, C.I.; QUINTERO, P.T.; AYALA, A.A.; ORTIZ, M.J. Drying characteristics of mango slices using the Refractance Window ${ }^{\mathrm{TM}}$ technique. Journal of Food Engineering, v. 109, n. 1, 2012, p. 69-75. https://doi.org/10.1016/j.jfoodeng.2011.09.032

OCHOA-MARTÍNEZ,C.I.;AYALA-APONTE,A.Modelos matemáticos detransferenciade masaen Deshidratación Osmótica. Ciencia y Tecnología Alimentaria, v. 4, n. 5, 2005, p. 330-342. https://doi.org/10.1080/11358120509487660

OLANIPEKUN, B.F.; TUNDE-AKINTUNDE, T.Y.; OYELADE, O.J.; ADEBISI, M.G.; ADENAYA, T.A. Mathematical Modeling of Thin-Layer Pineapple Drying. Journal of Food Processing and Preservation, v. 39, n. 6, 2015, p. 1431-1441. https://doi.org/10.1111/jfpp.12362

OLMOS, A. Cadena regional de piña departamento de Casanare. Yopal (Casanare): Gobernación de Casanare, Secretaria de Agricultura, Ganadería y Medio Ambiente, 2015, p. 1-15.

ONWUDE, D.I.; HASHIM, N.; JANIUS, R.B.; NAWI, N.M.; ABDAN, K. Modeling the Thin-Layer Drying of Fruits and Vegetables: A Review. Comprehensive Reviews in Food Science and Food Safety, v. 15, n. 3, 2016, p. 599-618. https://doi.org/10.1111/1541-4337.12196

ORTIZ-JEREZ, M.J.; GULATI, T.; DATTA, A.K.; OCHOA-MARTÍNEZ, C.I. Quantitative understanding of Refractance WindowTM drying. Food and Bioproducts Processing, v. 95, 2015, p. 237-253. https://doi.org/10.1016/j.fbp.2015.05.010

PONKHAM, K.; MEESO, N.; SOPONRONNARIT, S.; SIRIAMORNPUN, S. Modeling of combined far-infrared radiation and air drying of a ring shaped-pineapple with/without shrinkage. Food and Bioproducts Processing, v. 90, n. 2, 2012, p. 155-164. https://doi.org/10.1016/j.fbp.2011.02.008

POVEDA, N. Determinación de la influencia de las zonas de producción sobre el contenido de componentes bioactivos y la capacidad antioxidante de cinco frutas andinas [Tesis Ingeniería Bioquímica]. Ambato (Perú): Universidad Técnica de Ambato, 2014, p. 1-146. https://repositorio.uta.edu.ec/bitstream/123456789/8454/1/BQ\%2055.pdf

RAGHAVI, L.M.; MOSES, J.A.; ANANDHARAMAKRISHNAN, C. Refractance window drying of foods: A review. Journal of Food Engineering, v. 222, 2018, p. 267-275. https://doi.org/10.1016/j.jfoodeng.2017.11.032

RAJORIYA, D.; SHEWALE, S.R.; BHAVYA, M.L.; HEBBAR, H.U. Far infrared assisted refractance window drying of apple slices: Comparative study on flavour, nutrient retention and drying characteristics. Innovative Food Science and Emerging Technologies, v. 66, 2020, 102530.

https://doi.org/10.1016/j.ifset.2020.102530 
RANI, P.; TRIPATHY, P.P. Effect of ultrasound and chemical pretreatment on drying characteristics and quality attributes of hot air-dried pineapple slices. Journal of Food Science and Technology, v. 56, n. 11, 2019, p. 4911-4924. https://doi.org/10.1007/s13197-019-03961-w

SALAZAR, D.M.; ÁLVAREZ, F.C.; ACURIO, L.P.; PEREZ, L.V.; ARANCIBIA, M.Y.; CARVAJAL, M.G.;VALENCIA, A.F.; RODRIGUEZ, C.A. Osmotic concentration of pineapple (Cayenne lisse) as a pretreatment for convection drying. IOP Conference Series: Earth and Environmental Science, v. 292, n. 1, 2019. https://doi.org/10.1088/1755-1315/292/1/012039

SETHI, K.; KAUR, M. Effect of osmotic dehydration on physicochemical properties of pineapple using honey, sucrose and honey-sucrose solutions. International Journal of Engineering and Advanced Technology, v. 9, n. 1, 2019, p. 6257-6262. https://doi.org/10.35940/ijeat.A2026.109119

SHAKER, M.A. Comparison between traditional deep-fat frying and air-frying for production of healthy fried potato strips. International Food Research Journal, v 22, n. 4, 2015, p. 1557-1563.

SILVA, K.S.; GARCIA, C.C.; AMADO, L.R.; MAURO, M.A. Effects of Edible Coatings on Convective Drying and Characteristics of the Dried Pineapple. Food and Bioprocess Technology, v. 8, n. 7, 2015, p. 1465-1475. https://doi.org/10.1007/s11947-015-1495-y

SOUZA, D.G.; RESENDE, O.; DE MOURA, L.C.; JUNIOR, W.N.F.; ANDRADE, J.W.D.S. Drying kinetics of the sliced pulp of biofortified sweet potato (Ipomoea batatas L.). Engenharia Agrícola, v. 39, n. 2, 2019, p. 176-181. https://doi.org/10.1590/1809-4430-eng.agric.v39n2p176-181/2019

VEGA, A.; URIBE, E.; LEMUS, R.; MIRANDA, M. Hot-air drying characteristics of Aloe vera (Aloe barbadensis Miller) and influence of temperature on kinetic parameters. LWT-Food Science and Technology, v. 40, n. 10, 2007, p. 1698-1707. https://doi.org/10.1016/j.lwt.2007.01.001

VEGA-GÁLVEZ, A.; TELLO-IRELAND, C.; LEMUS-MONDACA, R. Simulación matemática del proceso de secado de la gracilaria chilena (Gracilaria Chilensis). Ingeniare. Revista Chilena de Ingeniería, v. 15, n. 1, 2007. https://doi.org/10.4067/S0718-33052007000100008

VILLAMIZAR, R.H.; QUICENO, M.C.; GIRALDO, G.A. Effect of vacuum frying process on the quality of a snack of mango (Manguifera indica L.). Acta Agronómica, v. 61, n. 1, 2012, p. 40-51.

WU, M.Y.; SHIAU, S.Y. Effect of the Amount and Particle Size of Pineapple Peel Fiber on Dough Rheology and Steamed Bread Quality. Journal of Food Processing and Preservation, v. 39, n. 6, 2015, p. 549-558. https://doi.org/10.1111/jfpp.12260

ZHANG, M.; CHEN, H.; MUJUMDAR, A.S.; TANG, J.; MIAO, S.; WANG, Y. Recent developments in high-quality drying of vegetables, fruits, and aquatic products. Critical Reviews in Food Science and Nutrition, v. 57, n. 6, 2017, p. 1239-1255. https://doi.org/10.1080/10408398.2014.979280

ZHANG, Z.; WEI, Q.; NIE, M.; JIANG, N.; LIU, C.; LIU, C.; LI, D.; XU, L. Microstructure and bioaccessibility of different carotenoid species as affected by hot air drying: Study on carrot, sweet potato, yellow bell pepper and broccoli. LWT Food Science and Technology, v. 96, 2018, p. 357-363. https://doi.org/10.1016/j.lwt.2018.05.061

ZZAMAN, W.; BISWAS, R.; HOSSAIN, M. A. Application of immersion pre-treatments and drying temperatures to improve comprehensive quality of pineapple (Ananas comosus) slices. Heliyon, v. 7, n. 1, 2021. https://doi.org/10.1016/j.heliyon.2020.e05882 